\title{
Caso Clínico: Tiroides Lingual Ectópica en Paciente Adulta Hipotiroidea. Diagnóstico Casual por Tomografía Computarizada.
}

*Correspondencia:

manuel.calvopina@udla.edu.ec

Teléfono [593] 23970000 Ext 2338

\section{Clinical Case: Ectopic Lingual Thyroid in Adult Hypothyroid Patient. Casual Diagnosis by Computed Tomography.}

\section{Pamela Tufiño Sánchez ${ }^{*}$, Daniela Silva Martinod1 ${ }^{i D}$, Clayreth Vinueza García1,2, Manuel Calvopiña Hinojosa1 $^{\text {iD }}$}

1. Universidad de las Américas, Facultad de Ciencias de Salud, Escuela de Medicina. Quito, Ecuador.

2. Medimágenes Cia Ltda. Red Complementaria de Salud, Quito-Ecuador.

\section{Resumen}

Introducción: Los casos de tiroides ectópica localizados en la base de la lengua son anormalidades congénitas raras y difíciles de diagnosticar. Razón de presentación del caso.

Caso Clínico: El caso corresponde a una mujer de 41 años con tiroides en base de la lengua diagnosticada incidentalmente con tomografía computarizada (TC), con antecedentes de hipotiroidismo y cáncer de mama derecha. Al examen físico de cuello no se palpa glándula tiroidea ni se observa masa o protuberancia en cavidad bucal. Por control del cáncer de mama, se solicita tomografía por emisión de positrones (PET) y ecografía de cuello, reportándose captación del radiofármaco en la región cervical anterior y superior de cuello, y ausencia de tejido glandular tiroideo a nivel habitual, respectivamente. Por cuanto, se realiza TC simple y contrastada observándose a nivel de la raíz de la lengua una imagen nodular hipercaptante que mide $23 \times 20 \times 20 \mathrm{~mm}$, bien definida, contornos regulares, no infiltra tejidos adyacentes, impronta luz de la orofaringe, sin individualizar la glándula tiroides a nivel habitual, corroborando así el diagnóstico de tiroides ectópica lingual.

Conclusión: El diagnóstico de tiroides ectópica en paciente adulto hipotiroideo es raro, por lo que debe considerarse la realización de TC si al examen físico y ecográfico no es palpable ni observable.

Palabras Claves: DISGENESIAS TIROIDEAS, TIROIDES LINGUAL, TOMOGRAFÍA COMPUTARIZADA PORRAYOS X, ECUADOR.

DOI: https://doi.org/10.33821/83
Copyright Tufiño, et al. Este artículo es distribuido bajo los términos de Creative Commons Attribution License 4.0 , el cual permite el uso y redistribución citando la fuente y al autor original. 


\begin{abstract}
Introduction: Cases of ectopic thyroid located at the base of the tongue are rare and difficult to diagnosecongenital abnormalities. Reason for presenting the case.

Clinical case: The case corresponds to a 41-year-old woman with thyroid based on the tongue diagnosed incidentally with computed tomography (CT), with a history of hypothyroidism and right breast cancer. On the physical examination of the neck, the thyroid gland is not palpated, and no mass or bump is observed in the oral cavity. For breast cancer control, positron emission tomography $(\mathrm{PET})$ and neck ultrasound are requested, radiopharmaceutical uptake is reported in the anterior and upper cervical neck region, and absence of thyroid glandular tissue at the usual level, respectively. As a simple and contrasted CT scan, a hypercapting nodular image measuring $23 \times 20 \times 20 \mathrm{~mm}$, well defined, regular contours is observed at the root of the tongue, does not infiltrate adjacent tissues, oropharynx light imprint, without individualizing the thyroid gland to usual level, thus corroborating the diagnosis of lingual ectopic thyroid.
\end{abstract}

Conclusion: The diagnosis of ectopic thyroid in an adult hypothyroid patient is rare, so CT should be considered if the physical and ultrasound examination is not palpable or observable.

Keywords: THYROID DISGENESIES, LINGUAL THYROID, X-RAY TOMOGRAPHY, ECUADOR.

\section{Introducción}

La tiroides es una glándula endocrina ubicada en la parte anterior del cuello, profunda en relación a los músculos esternocleidomastoideo y esternohioideo, compuesta por 2 lóbulos, izquierdo y derecho, de ubicación anterolateral a la tráquea y laringe [1,2]. Si existe alteraciones en el descenso tiroideo, se puede encontrar tejido glandular aberrante a lo largo del trayecto del conducto tirogloso. La ectopia tiroidea es un tipo de disgenesia con una prevalencia de 1:7.000, siendo más común en mujeres que en hombres con una relación 4:1 [3,4]. Se describen ectopias linguales, sublinguales, tiroglósicas e intralaringotraqueales, siendo las primeras las más frecuentes [5].

Si la tiroides ectópica lingual es observable, se puede evidenciar una masa rosada, de superficie lisa y firme en la línea media de la base de la lengua, de aspecto vascularizado. El $70 \%$ de los pacientes presenta la glándula contenida enteramente en la base de la lengua. El $10 \%$ de pacientes es asintomático y del 33 al $70 \%$ presentan sintomatología hipotiroidea [6]. Algunas tiroides ectópicas linguales se manifiestan con cuadros obstructivos con disfagia y disfonía, hemorragia y función endócrina normal o alterada [1]. Se precisa realizar diagnóstico diferencial con hemangiomas, linfomas, fibromas, abscesos y tumores malignos de la lengua [7]. Casos de tiroides ectópica lingual son diagnosticados de forma incidental con métodos de imagen $[3,6]$.

En el diagnóstico de tiroides ectópica lingual los exámenes de imagen son fundamentales para determinar la localización exacta, tamaño y estructura de la glándula. La gammagrafía tiroidea con isótopos radioactivos (1123, 1131 y Tc99) es la prueba más específica porque permite la evaluación de la captación y la funcionalidad de la glándula fuera de su lugar habitual; sin embargo, es costosa, radioactiva y está indicada en casos donde la ecografía o la tomografía computarizada (TC) no muestran la glándula tiroidea en su situación habitual 
[2, 3, 8]. El ultrasonido cervical indica ausencia de glándula tiroides y el de alta resolución favorece la exploración especialmente en pacientes que presentan masas en el cuello; su sensibilidad aumenta si se utiliza con técnica Doppler para demostrar señales de flujo sanguíneo periférico o internos que reflejan hipervascularidad [8, 9].

Por otro lado, la tomografía computarizada simple determina el tamaño glandular, el tejido tiroideo muestra una mayor densidad que los tejidos blandos circundantes en la fase simple; adicionalmente, la TC contrastada permite localizar anatómicamente masas cervicales de forma precisa cuando donde no se ha podido determinar ecográficamente [8]. La resonancia magnética $(R M)$ puede ser de utilidad si se requieren imágenes más específicas o en casos donde la delimitación del tejido tiroideo del músculo lingual sea difícil, siendo la intensidad del tejido tiroideo normal mayor que el muscular en las secuencias $\mathrm{T} 1$ y $\mathrm{T} 2$ [8-10].

Debido a la rareza de esta patología se presenta el caso clínico.

\section{Caso Clínico}

Mujer de 41 años, con antecedentes de cáncer de mama derecha e hipotiroidismo en tratamiento con levotiroxina $100 \mathrm{mcg}$ vía oral en ayunas cada día por 2 años. Al momento del estudio con TSH $10.73 \mathrm{UI} / \mathrm{mL}$ (rango normal: $0.7-4.20$ ), FT3: $4.23 \mathrm{pg} / \mathrm{mL}$ (rango normal: 2.00- 4.40), FT4: $1.05 \mathrm{ng} / \mathrm{dL}$ (rango normal: 0.93-1.70). No refiere sintomatología, evidenciando asíhipotiroidismo subclínico.

Al examen físico de cuello no se palpa glándula tiroidea ni se evidencia alguna masa o protuberancia en región cervical ni en cavidad bucal. Por el cáncer de mama recibió quimioterapia y radioterapia, con un total de seis ciclos hace 2 años; en la actualidad recibe hormonoterapia con controles anuales.

En el último control, se solicita una tomografía con emisión de positrones (PET) que reporta captación del radiofármaco en la región cervical anterior y superior. Con este resultado, en el servicio de oncología se solicita ecosonografía de cuello que reporta "ausencia de tejido tiroideo pretraqueal, elementos vasculares de características normales, 5 formaciones ganglionares cervicales derechas no mayores a $18 \mathrm{~mm}$, de aspecto reactivo-inflamatorio, la mayor de ellas ubicada en el nivel IIA. En región cervical izquierda, nivel III, se evidencian 6 ganglios de aspecto reactivo-inflamatorio de hasta $9.5 \mathrm{~mm}$. Glándulas submaxilares sin alteraciones" (Figura 1). 
Figura 1. Ecografía de tejidos blandos de Cuello

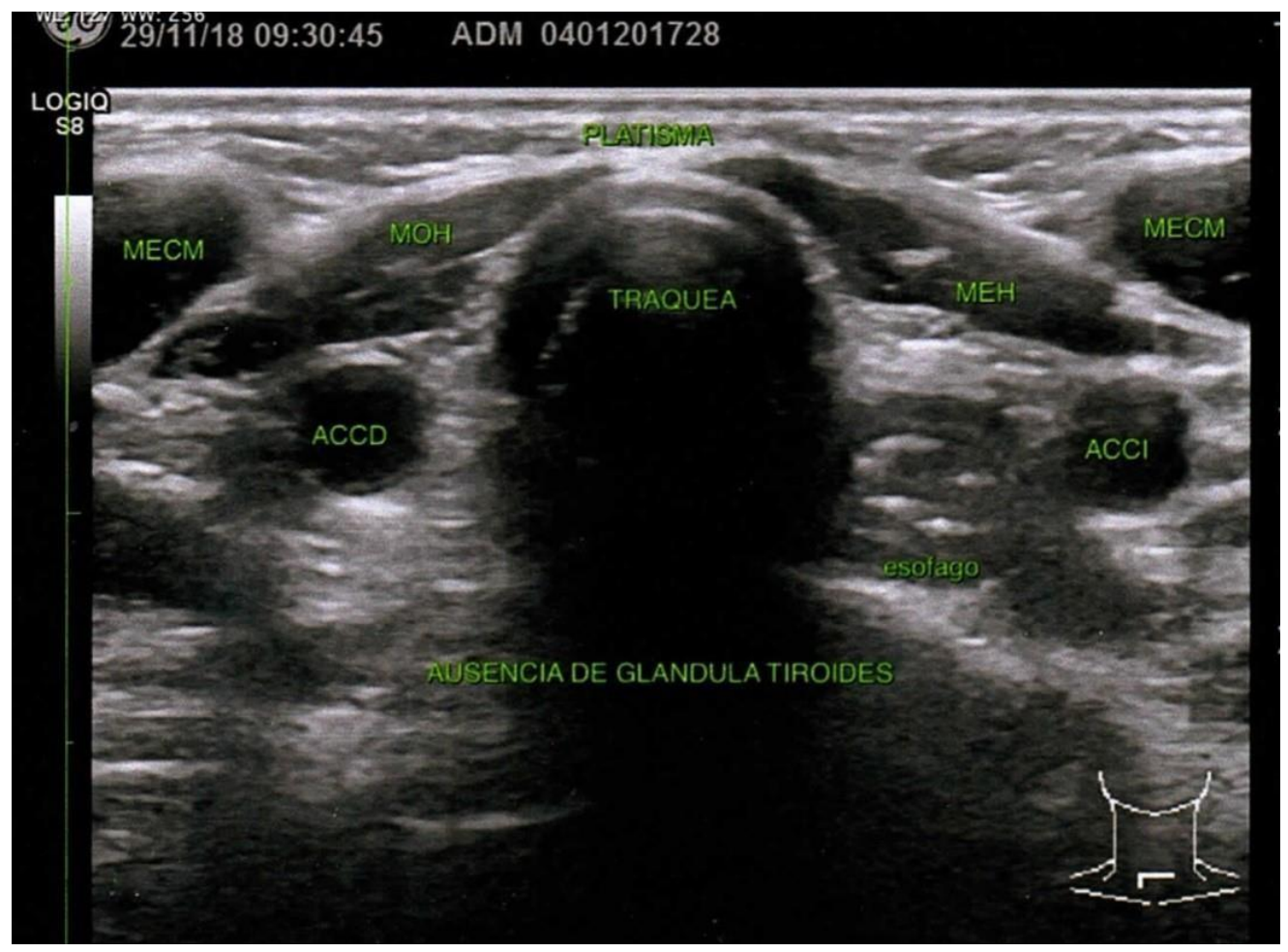

Imagen ecográfica de cuello. En esta imagen no se observa tejido tiroideo en la parte anterior del cuello anterolateral a la tráquea. Los elementos vasculares tienen características normales.

Se realiza TC simple y contrastada que reporta "A nivel de la raíz de la lengua, en la línea media, se observa una lesión hipodensa en fase simple, con realce importante luego de la administración de contraste intravenoso, nodular, mide $23 \times 20 \times 20 \mathrm{~mm}$, bien definida, contornos regulares, no infiltra tejidos adyacentes, impronta la luz de la orofaringe. Antros maxilares neumatizados, fosas pterigoides libres, elementos musculares del cuello de características normales, simétricos. Las estructuras vasculares del cuello demuestran adecuada morfología, no defectos de llenado; no existen conglomerados adenomegálicos cervicales. A nivel de la hipofaringe y laringe no se advierte patología. Glándulas parótidas de características normales. Glándulas submaxilares, simétricas. No se evidencia tejido tiroideoa nivel habitual" (Figura 2). 
Figura 2. Tomografía de cuello

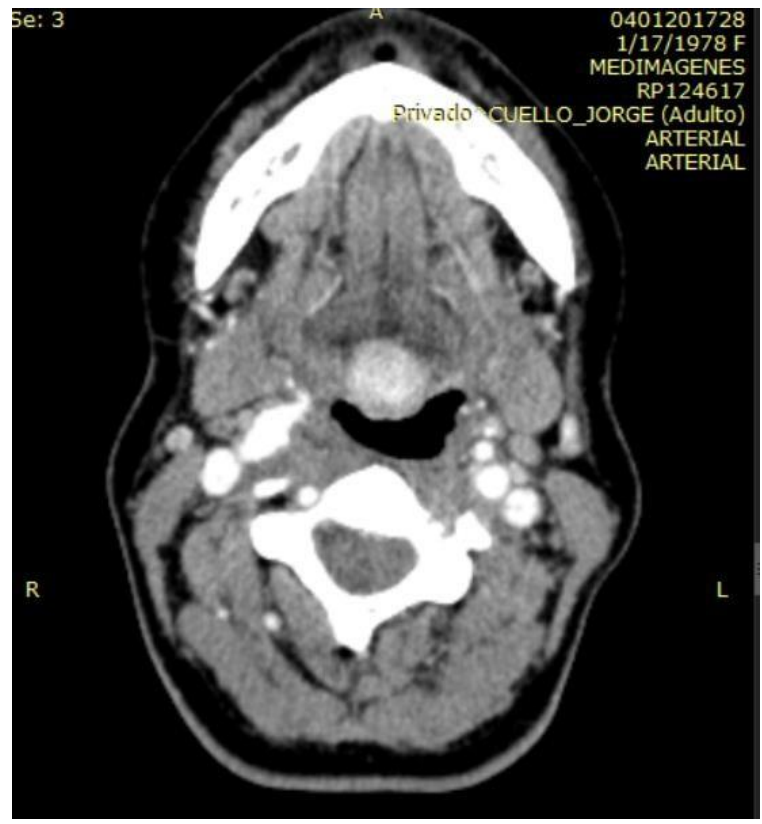

Imagen tomográfica contrastada/ fase arterial. A nivel de la raíz de la lengua, en la línea media, se observa

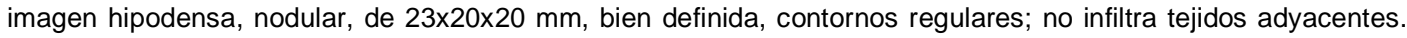
No se individualiza la glándula tiroides a nivel pretraqueal".

Con estos resultados y en correlación con la clínica se confirma hipotiroidismo subclínico y con los resultados de imagen de TC simple y contrastada se diagnostica tiroides ectópica enbase de la lengua.

\section{Discusión}

Hasta donde conocemos y por revisiones de la literatura realizadas, este es el primer caso reportado en Ecuador de ectopia lingual de la glándula tiroides. En la paciente nunca se sospechó de ectopia lingual tiroidea, casualmente luego del PET por control de su cáncer de mama que reporta captación del radiofármaco en la región cervical anterior y superior, se presume la patología. Mientras que la ecosonografía observa ausencia de tejido tiroideo pretraqueal, la TC reporta lesión hipodensa, nodular, de tamaño 23×20×20 mm, bien definida y de contornos regulares, siendo definitivo el diagnóstico de tiroides ectópica lingual. No fue necesario realizar RM o gammagrafía tiroidea con isótopos radioactivos. Los métodos de imagen son importantes en el diagnóstico de tiroides ectópicas [2, 11,12]. La presentación de tiroides ectópicas son anormalidades congénitas raras y difíciles de diagnosticar; en el presente caso, el diagnóstico fue confirmado por TC. Muchos de los casos son asintomáticos [2], como ocurrió en este reporte, que al examen físico de cuello no se palpó glándula tiroidea ni se observó masa en cavidad bucal; otros se presentan con disfagia, disfonía, voz gangosa, entre otros síntomas que se han reportado previamente [11-12]. 
La localización lingual de la glándula tiroides está entre las ectopias más frecuentes, ocurriendo en el $90 \%$ de casos [1, 8], como en el presente reporte.

Hasta el $70 \%$ de pacientes con tiroides ectópica presentan síntomas de hipotiroidismo [3, 6]. Esta paciente fue diagnosticada de hipotiroidismo desde hace 2 años, en tratamiento con levotiroxina. Por los valores actuales de TSH y T4 presenta hipotiroidismo subclínico. Estas ectopias son diagnosticadas especialmente en la niñez 0 adolescencia, pero en este caso fue en la edad adulta, indicando la falta de conocimiento sobre la presencia de esta patología y la realización de exámenes de imagen para su confirmación. Otros casos fueron diagnosticados casualmente por síntomas como disfagia u orofaríngeos [3].

En el diagnóstico por imagen de tiroides ectópica lingual, la literatura indica que, en la ecosonografía de cuello, no se evidenciará tejido tiroideo en su localización habitual; en cambio en la TC se observará una masa encapsulada multiloculada en la línea media con presencia de realce al momento de colocar contraste [10], como ocurrió en el presente caso.

Se descarta un carcinoma tiroideo metastásico ya que, en el ultrasonido se hubiese visualizado una lesión hipoecogénica con linfadenopatía cervical concurrente; mientras que, en la TC, una masa no bien definida, con posibilidad de gran extensión y un realce heterogéneo[13].

Si se sospechase de un carcinoma de células escamosas, las características en la TC serían las de una masa heterogénea y realzada en la base de la lengua de ubicación lateral o medial,junto con linfadenopatía asociada [13].

Por otro lado, en caso de un absceso tiroideo, en el eco se evidenciaría una masa complicada con restos internos, ecogenicidad heterogénea y vascularizada; además, en la tomografía, una densidad heterogénea con realces heterogéneos y linfadenopatía [13].

\section{Conclusiones}

En conclusión, ante la ausencia de masa tiroidea al examen físico de cuello en una paciente hipotiroidea y la no observación ecosonográfica de la glándula en su localización habitual pretraqueal o infrahioidea, la realización de la TC es fundamental para visualizar las características de la glándula tiroides en lugares fuera de su ubicación normal.

\section{Agradecimientos}

Se reconoce a las personas que participaron indirectamente en el caso clínico. Adicionalmente queremos reconocer al paciente que autorizó la publicación del caso con fines académicos. 


\section{Información adicional}

Abreviaturas

TC: Tomografía Computarizada.

PET: Tomografía por emisión de positrones.

Nota del Editor

La Revista Oncología Ecu permanece neutral con respecto a los reclamos jurisdiccionales en mapas publicados $y$ afiliaciones institucionales.
Archivos Adicionales

Ninguno reportado.

\section{Fondos}

Los fondos para la presente investigación fueron propios de los autores del presente artículo.

\section{Disponibilidad de datos y materiales}

Existe la disponibilidad de datos bajo solicitud al autor de correspondencia. No se reportan otros materiales.

\section{Contribuciones de los autores}

PTS, DSM, CVG, realizaron la revisión bibliográfica y compilación de la historia clínica. $\mathrm{MCH}$ realizó la interpretación radiográfica y escritura del artículo. PTS y $\mathrm{LMCH}$ realizaron el análisis crítico del artículo. Todos los autores leyeron y aprobaron el manuscrito final.

\section{Aprobación de ética y consentimiento para participar}

No aplica a un estudio de caso.

\section{Consentimiento para publicación}

Los autores disponen el consentimiento firmado por parte del paciente para la publicación del caso clínico con fines académicos. 
Manuel Calvopiña Hinojosa, Médico graduado en la Universidad Central del Ecuador, phd en Ciencias Médicas, Universidad de KochiJapón.

\section{Referencias}

Abreviaturas en la referencias

\section{DOI: Digital Object Identifier}

PMID: PubMed Identifier SU: Short URL
1. Askin F, Rosai J, Sibley R, Dehner L, McAlister W. Malignant small cell tumour of the thoracopulmonary region in childhood: a distinctive clinicopathologic entity of uncertain histogenesis. Cancer. 1979;43(6):2438-51.

2. Martínez A, Cano I, Carrillo M, Ruiz J. Tumor neuroectodérmico primitivo de la pared torácica en adulto. Presentación de un caso. An Radiol México. 2011; 10(1):43-47.

3. Benbrahim Z, Arifi S, Daoudi K, Serraj M, Amara B, Mohammed Ch. Askin's tumor: a case report and literature review. Wold J Surg Oncol. 2013; 11:10.

4. Gómez C, Ramírez S, Quesada D, Unigarro L. Tumor de Askin: Presentación de caso. Rev Colombi Radiol.2011;22(4):3378-81.

5. Iribarren $M$, Carnerero $V$, Domínguez $P$, González $R$. Tumor neuroectodérmico primitivo (tumor de Askin) en la pared torácica. An Pediatr (Barc). 2011; 75(5):343-344.

6. Bravo D, Hernández $K$, Linares $A$, Jaramillo $L$, Méndez $M$, Restrepo $F$, et al. Tumor neuroectodérmico primitivo de la pared torácica: tumor de Askin en niños. Rev Fac Med. 2013; 61(3):239-244.

7. Shrestha B, Kapur B, Karmacharya K, Kakkar S, Ghulianis R. Askin's Tumor: A Dual Case Study. Inter J Pediatr. 2011; 2011: 252196. DOI: 10.1155/2011/252196.

8. Suárez M, Osorio M. Tumor de Askin: Presentación de un caso y revisión de la literatura. An Radiol México. 2008; 1:55-60.

9. Dou X, Yan H, Wang R. Treatment of an Askin tumor: A case report and review of the literature. Oncol lett. 2013; 6(4):985-989.

10. Moujahid M, Ziadi T, Rhari A, Nador K, Serghini I, Tahiri M. The Rare Thoracic Tumor, Askin Tumors 7 Cases Report. Open J Thoracic Surg. 2014. 4: 27-31. 